\title{
Finding the hot spot: identifying immune sensitive gastrointestinal tumors
}

\author{
Andre Luiz Pitanga Bastos De Souza \\ Lifespan Cancer Institute, Research Office APC room 131, 593 Eddie Street, Providence, RI 02905, USA \\ Correspondence to: Andre Luiz Pitanga Bastos De Souza. Lifespan Cancer Institute, Research Office APC room 131, 593 Eddie Street, Providence, RI \\ 02905, USA. Email: andre.desouza@lifespan.org.
}

\begin{abstract}
Although researchers have been trying to harness the immune system for over 100 years, the advent of immune checkpoint blockers (ICB) marks an era of significant clinical outcomes in various metastatic solid tumors, characterized by complete and durable responses. ICBs are monoclonal antibodies that target either of a pair of transmembrane molecules in tumors or T-cells involved in immune evasion. Currently 2 ICBs targeting the checkpoint program death 1 (PD-1), nivolumab and pembrolizumab, and one cytotoxic lymphocyte antigen-4 (CTLA-4) inhibitor (ipilimumab) are approved in gastrointestinal malignancies. We review herein the current evidence on predictive biomarkers for ICB response in gastrointestinal tumors. A review of literature based on the National Cancer Institute list of FDA-approved drugs for neoplasms and FDA-approved therapies at the FDA website was performed. An initial literature review was based on the American Association for Clinical Research meeting 2019, the American Society of Clinical Oncology meeting 2019 and the European Society of Medical Oncology 2019 proceedings. A systematic search of PubMed was performed involving MeSH browser terms such as biomarkers, immunotherapy, gastrointestinal diseases and neoplasms. When appropriate, American and British terms were used in the search. The most relevant predictor of response to ICBs is microsatellite instability (MSI) and the data is strongest for colorectal cancer. At least 3 prospective trials show evidence of PD-L1 as a predictive biomarker for ICB response in gastroesophageal malignancies. At least one prospective trial has described tumor mutational burden high (TMB-H), independent of MSI, as predictive of response in anal and biliary tract carcinomas. DNA Polymerase Epsilon (POLE) or delta (POL-D) mutations have been implicated in a subset of MSS colorectal cancer with TMB-H but this biomarker requires prospective validation. There is evolving data based on retrospective observations that gene alterations predicting acquired resistance and hyper-progression. Ongoing clinical research is assessing the role of the human microbiome and RNA-editing complex mutations as predictive biomarkers of response to ICBs. MSI has the strongest predictive power among current biomarkers for ICB response in gastrointestinal cancers. Data continue to accumulate from ongoing clinical trials and new biomarkers are emerging from pre-clinical studies, suggesting that drug combinations targeting pathways complimentary to the PD-1/PD-L1 axis inhibition will define a robust field of clinical research.
\end{abstract}

Keywords: Biomarkers; gastrointestinal diseases; immunotherapy; neoplasms

Received: 07 August 2019; Accepted: 06 December 2019; Published: 05 October 2020.

doi: $10.21037 / \operatorname{tgh} .2019 .12 .11$

View this article at: http://dx.doi.org/10.21037/tgh.2019.12.11 


\section{Introduction}

Although researchers have been trying to harness the immune system for 100 years starting with Coley experiments with Streptococcus, the advent of immune checkpoint blockers marks a watershed in immune therapy research characterized by complete and durable responses in a significant proportion of patients with metastatic solid tumors. Immune checkpoint blockers (ICB) are monoclonal antibodies that target either of a pair of transmembrane molecules in tumors or T-cells involved in immune evasion. The currently approved ICBs include programmed death 1 and its ligand (PD-1 and PD-L1) inhibitors and the cytotoxic lymphocyte antigen-4 (CTLA-4) antagonist ipilimumab. Herein, we review the current evidence on biomarkers for ICB response in gastrointestinal tumors.

Lynch was the first to describe a genetic syndrome in a subset of patients with concurrent gastrointestinal malignancies. It is now known that Lynch syndrome represents $4 \%$ of colorectal cancers. Later, the syndrome was attributed to defective proteins involved in gene repair that resulted in genomic instability in segments of DNA called microsatellites. It has been demonstrated that microsatellite instability imparts a high TMB to its carriers. By hypothesizing that high TMB would translate into high neoantigen burden, a series of clinical trials showed that patients with gastrointestinal tumors with high microsatellite instability refractory to standard chemotherapy derive significant clinical benefit from ICBs. Molecularly, microsatellites are triplet base pair repeats that occur throughout the DNA currently measured in next generation sequencing platforms in specific segments of the genome (46 loci, for example). The discordance rate between IHC and PCR for detecting MSI is less than $5 \%$ and IHC is less reliable and sensitive than PCR-based testing $(1,2)$. Besides both tests are validated for colorectal and endometrial cancer while it is not for other MSI-related malignancies (conspicuously ovarian cancer, where the IHC/PCR concordance rate is only $68 \%$ ) (3).

TMB was theorized to be an indirect determinant of neoantigen burden, which is thought to be associated with an effective T-cell response on immune checkpoint inhibition. A study has described the frequency of TMB in various gastrointestinal malignancies (4). TMB was measured by counting all nonsynonymous missense mutations not previously described as germline alterations in a 1.4 Megabase sequence of biopsied tumor in a commercial next generation sequencing platform. High
TMB was defined as more than 17 mutations per Megabase. There will be allusions to this study throughout this text in the histology sections. Also, it will be shown here the data from a prospective study of pembrolizumab pre-specifying $\mathrm{TMB}$ as a biomarker in solid tumors including separate cohorts for anal squamous cell carcinoma (SCC) and biliary tract carcinoma.

The programmed death ligand 1 expression has been standardized for each of the immune checkpoint inhibitors. Some of these PD-L1 assays evaluate membrane expression only in tumor cells (TC) while others measure it on lymphocytes and macrophages [immune cells (IC)] as well. The correlation of PD-L1 expression is not only histology specific but depends on the ICB used for the study evaluating an assay as a biomarker. The value of PD-L1 as an immune checkpoint inhibitor predictive biomarker is strongest in esophageal tumors. In a study describing MSI, TMB and PD-L1 across gastrointestinal malignancies, incongruent PD-L1 positivity and TMB-H were seen in right-sided colon cancer and small bowel adenocarcinomas (low rate of PD-L1 positivity and high rate of TMB-H) and in GISTs, anal and esophageal squamous cancers (high rate of PD-L1 positivity and low rate of TMB-H). PD-L1 positivity is more likely to be seen in MSI than MSS tumors (4).

\section{Gastroesophageal cancer}

Microsatellite instability is correlated with poor prognostic features in some, but not all gastrointestinal malignancies. The prevalence of MSI is gastroesophageal cancers is $1.5 \%$ (5). Although some studies have linked MSI-H in gastric cancer to survival (6-13), other have failed to demonstrate any benefit (14-18). Remarkably, Kim et al. showed MSI-H defines a good prognostic group in patient with intestinal type gastric but imparts a poor prognosis for diffuse type gastric cancer (6).

Expanding not only on the role of tumor mutational burden (TMB) but also of MRD and PD-L1 expression as predictive biomarkers to immune checkpoint inhibitors response in gastrointestinal tumors, investigators performed a 592 gene panel next generation sequencing of 4,125 specimens from 14 distinct cancers (4). High TMB was defined as more than 17 non-germline nonsynonymous missense mutations per megabase. Deriving data from 2,000 matching specimens to achieve a sensitivity of more than $95 \%$ and specificity of more than $99 \%$, MSI was specified as 46 or more loci with insertions or deletions. PD-L1 positivity was established as a moderate or strong 
staining on SP142 primary antibody. Mean age was 61 years (range, 12-90 years), 59\% of specimens were from primary tumors and $37 \%$ from metastatic sites. Primary tumors had a greater TMB-high rate than metastatic sites $(5.7 \% v s$. $3.0 \%)$. Gastric adenocarcinomas had an intermediate rate of TMB-high (8.3\%). The rate of TMB-H was noticeable different between gastric cancers and gastroesophageal adenocarcinomas ( $8.3 \%$ vs. $3.1 \%)$ (4).

Investigators evaluated 160 patients with locally advanced esophageal SCC who underwent esophagectomy and showed that PD-L1 but not PD-L2 overexpression was an independent prognostic factor for DFS (HR: 1.713; 95\% CI, 1.020-2.880; $\mathrm{P}=0.042$ ) (19). Aside statistical issues with multiplicity due to numerous pre-specified subgroups and arms (20), a trial investigated clinical outcomes in three therapy arms (pembrolizumab with combined fluoropyrimidine and platinum, combined chemotherapy alone and pembrolizumab alone) with PD-L1 combined (tumor and immune cells) positive (to 2 cut-offs $>1 \%$ and $>10 \%$ ) score (CPS) as biomarker. The only pre-specified subgroup to derive benefit from pembrolizumab alone versus chemotherapy were patients with CPS 10 or higher, achieving a 6.6 median overall survival benefit (HR 0.69; 95\% CI, 0.49-0.96) (21). Based on 35 patient samples, Derks' group described that $42 \%$ of Barret's esophagus (but none of other types of esophagitis) overexpress PD-L2 (22), although we lack definitive data on PD-L2 as a biomarker in gastroesophageal cancers.

Gastric adenocarcinoma was classified by The Cancer Genome Atlas in 4 molecular subgroups, with two subgroups (Epstein-Barr virus signature and microsatellite unstable) proposed as biomarker contenders for higher responses to checkpoint inhibition. In gastric adenocarcinoma, Epstein-Barr virus infection is associated with $9 \mathrm{q}$ chromosomal amplification that leads to upregulation of PD-L1 and PD-L2. Pembrolizumab was approved for metastatic gastric cancer refractory to at least 2 lines of standard-of-care therapy (and Her2neu therapy when appropriate) with a CPS PD-L1 of at least $1 \%$ based on a $57 \%$ response rate and duration of response up to 14 months $(23,24)$. Only $3 \%$ of the patients of that cohort had MSI. Based on a promising overall response rate of $20 \%$ in a phase 2 trial in patients with esophageal cancers, pembrolizumab was compared with chemotherapy of choice (paclitaxel, docetaxel or irinotecan) in 628 randomized patients stratified by histology (squamous or adenocarcinoma), showing a median overall survival benefit of 2.6 months $(25,26)$. Pembrolizumab is now approved for esophageal SCC with a CPS higher than $10 \%$.

\section{Pancreatic ductal adenocarcinoma}

Pancreatic adenocarcinoma is considered an immuneresistant tumor due to the mechanical barrier provided by the desmoplastic tumor microenvironment. Only $0.2 \%$ of pancreatic ductal adenocarcinomas are MSI-H (4). No studies assessing MSI as a prognostic factor in pancreatic ductal adenocarcinoma (27-29) have reached statistical significance to date. Ipilimumab was investigated in 23 patients with pancreatic ductal adenocarcinoma (PDAC) with no objective response (30). In a trial of the IgG4 monoclonal antibody targeting PD-L1 BMS 936559, which included 14 patients with PDAC, none had an objective response (31). The same results had been achieved in a retrospective analysis of patients with solid tumors given atezolizumab which included 5 patients with PDAC (32). Currently several combinations of chemotherapy (gemcitabine and ipilimumab, nab-paclitaxel and nivolumab), radiotherapy (with nivolumab, the PD-L1 inhibitor durvalumab and the CTLA-4 inhibitor tremelimumab) and vaccines (GVAX and nivolumab, GVAX, CRS-207 and nivolumab and CRS207, nivolumab and ipilimumab) are ongoing. Among other strategies to overcome tumor microenvironment immune resistance include combination of atezolizumab with hyaluronidase and downregulation of stromal promoters through the TGF-beta inhibitor galunisertib with gemcitabine and the TGF receptor 2 inhibitor trabedersen, which rendered one complete response $(33,34)$. Other studies investigated irreversible electroporation associated with PD-1 blockade, histone deacetylase inhibitors promoting downregulation of myeloid derived suppressor cells, and oncolytic virus (with 2 documented partial responses) (35). Clinical trials were launched based on successful pre-clinical data of chimeric antigen receptor adoptive T-cell therapy targeting mesothelin in PDAC $(36,37)$. While Beatty et al documented stable disease by metabolic activity measurements in 3 out 6 patients with pancreatic adenocarcinomas treated with mesothelintargeted CAR-T cells (38), respiratory toxicity due to preconditioning has limited efficacy in CEA-targeted CAR-T cells (39). A myriad of clinical trials involving CAR-T cells with distinct targets are recruiting patients around the world.

\section{Hepatocellular carcinoma (HCC)}

As HCC is mostly driven by a chronic viral infection 
consistently coping with the host immune system, it was hypothesized that immune invasion plays a role on HCC progression. A research team working exclusively with 755 patients with advanced HCC recently reported a median TMB of 4 mutations/Mb with only 6 tumors $(0.8 \%)$ found to be TMB-high. Out of 542 cases assessed for microsatellite instability (MSI), one $(0.2 \%)$ was MSIhigh and TMB-high. Although 27 (4\%) patients had DNA polymerase Epsilon or delta (POLE/D) alterations, the one patient who had a pathogenic POLE R762W mutation had TMB was 4 mutations/Mb. Forty percent had DNA damage response gene alterations. One patient (TMB 15 mutations/Mb, MSI-low) out of 17 who was known to the center from the dataset had a durable complete response to nivolumab lasting more than 2 years (40).

The accelerated approval of nivolumab for patients with HCC independent of hepatitis B or C status added one more second line option after sorafenib based on a phase 2 trial that demonstrated an ORR of $13.3 \%$, CR of $1.4 \%$. In this trial, $91 \%$ of patients with ORR maintained response at 6 months and 55\% continued to respond after 12 months (41). The next step was the accelerated of pembrolizumab after results from the phase 2 trial (where 64\% of 104 patients had metastases), reaching an ORR of $17 \%$, a complete response rate of $1 \%, 89 \%$ 6-month duration of response, and $56 \%$ twelve-month duration of response (42). While the follow-up phase 3 trials of these accelerated approvals (a demand of this FDA mechanism) comparing pembrolizumab with placebo after first line sorafenib and nivolumab with sorafenib in the first line setting failed to reach statistically significant results, biomarkers or combination approaches may refine the subsets of patients who may benefit from immune oncology (IO) drugs in these settings $(43,44)$. Of note, one the first prospective efforts to define tumor mutation load as a predictive biomarker included 3 patients with HCC, a number too low to derive any conclusions at this point (45). A meta-analysis of 16 studies incorporating 3,533 patients concluded that high PD-L2 expression correlates to poor disease free survival in patients with HCC who undergo curative resection $(\mathrm{HR}=1.44 ; 95 \% \mathrm{CI}, 1.15-1.81 ; \mathrm{P}=0.001)$, but not to overall survival (46).

\section{Biliary tract cancer}

A MSI prevalence of $2.3 \%$ among hepatobiliary cancers was previously described (5). No studies assessing MSI as a prognostic factor in gallbladder (47) or extrahepatic biliary duct carcinoma (46) have reached statistical significance to date. Ruemelle and collaborators found MSI was prognostic of survival in ampullary carcinoma (48). A high TMB rate (at threshold of 15 mutations/Mb) was shown only $2.9 \%$ of patients with biliary tract cancer (49). Interestingly, distinct origins (biliopancreatic versus intestinal) of ampula of Vater tumors may define distinct susceptibility to immune checkpoint inhibitors (50).

\section{Small bowel adenocarcinoma}

MSI high is present in $8.6 \%$ of small bowel adenocarcinomas (4). Small-bowel adenocarcinoma was shown to have one of the highest prevalence of TMB high tumors $(10.2 \%)$ and one of the highest average TMB (10.2 mutations/MB), second only to right-sided colon adenocarcinomas (5). A discrepancy rate of high TMB between primary and metastatic site was observed in small bowel adenocarcinoma (14.4\% vs. $3.7 \%$ ) (5). MSI was found to be a good prognostic factor, and was associated with a longer cancer specific survival (51). The molecular pathology of small cell carcinoma is distinct when associated with celiac disease, and data is missing about response or gastrointestinal toxicity of immune checkpoint inhibitor in this auto-immune disease $(52,53)$. The Vanderbilt University is currently recruiting patients with small bowel adenocarcinoma for a study of avelumab (NCT03000179), while a multicentric study involving almost 900 cancer centers continues to investigate the combination of nivolumab and ipilimumab among 53 rare tumors, including small bowel adenocarcinoma (NCT02834013). Biomarker analysis from these two trials are eagerly awaited.

\section{Colorectal adenocarcinoma}

Microsatellite instability is described in $15 \%$ of patients with nonmetastatic and $5 \%$ of patients with metastatic colon adenocarcinoma (54-56). Historically, patients with microsatellite unstable (MSI) colorectal cancer (CRC) were the first to derive clinical benefit from ICBs. Before MSI was the most validated biomarker of immune checkpoint inhibitors in CRC, it was a prognostic factor and a predictor of response to certain chemotherapy agents. MSI was the strongest predictor of relapse in a population of Italian patients with T3N0M0 CRC (57). A pooled analysis study involving seven prospective studies of adjuvant therapy in CRC investigated the survival after recurrence (SAR) of 2,630 patients who presented with stage III CRC, finding that MSI/MRD had a significantly longer SAR 
than MSS/MMR after multivariate analysis (58). A single center study concluded that MMR is not a predictor of progression free survival or overall survival after CAPOX or FOLFOX therapy in patients with recurrent or metastatic CRC patients from South Korea (59). Two thirds of MSI in advanced colorectal tumors are due to promoter methylation of MLH1 and it is unknown if this epigenetic change affect responsiveness to ICBs (60). Of note, the MSI prevalence in appendiceal carcinomas was found to be $3 \%$ in a single center series of 108 cases, with rare MLH1 promoter methylation (61).

Investigators define the correlation between MSI by Immunohistochemistry and clinic characteristics. Loss of expression of all four immunohistochemical markers (MLH-1, MSH2, PMS-2, MSH-6) was correlated with poorly differentiated and mucinous adenocarcinoma histology $(\mathrm{P}<0.0001, \mathrm{P}=0.015, \mathrm{P}<0.0001, \mathrm{P}<0.0001$ respectively). Loss of MLH-1 and PMS-2 expression was correlated with poor lymphovascular invasion $(\mathrm{P}=0.032$ and $\mathrm{P}<0.0001$ ), a poor prognostic factor in stage II CRC (62). MSI is also a predictor of lymph node yield at time of hemicolectomy for CRC (63). A study showed that dMMR was a negative predictor for 5-FU efficacy in the preoxaliplatin era, showing even reduced overall survival in patients with Stage II CRC (64). Investigators demonstrated that MSI is not a predictor of disease-free survival or overall survival in patients with colorectal adenocarcinoma treated with adjuvant FOLFOX (65). Upregulation of PD-L1 by chemoradiation in nonmetastatic rectal adenocarcinoma was found to be a poor prognostic factor and may be a setting for combinations with immunotherapy such as the European NCT03127007, combining chemoradiation with atezolizumab (66).

Pembrolizumab was approved for patients with MSI based on a study of 149 patients ( 90 with CRC) involving 15 different cancers, reaching an overall response rate (ORR) of $39.6 \%$ and a 6-month duration of response of $78 \%$ (67). This was followed by a study enrolling 74 patients with advanced CRC to a single nivolumab arm after at least one line of therapy, which reached a $31.1 \%$ ORR, a 12 -month disease control rate (DCR) of $69 \%$, and a 12 -month duration of response of $86 \%$ among responders, leading to the approval of Nivolumab for metastatic cancers with microsatellite instability after standard line therapy independent of histology (68). A cohort of 82 patients with metastatic CRC refractory to 2 standard of care lines of therapy and treated with nivolumab and ipilimumab achieved an ORR of $46 \%$ (and 3 complete responses), a 12-month progression free survival (PFS) of $71 \%$, and a 12 month overall survival (OS) of 85\%, warranting its approval (69).

Right-sided colon adenocarcinomas were shown to have the highest prevalence of TMB high tumors (14.6\%) and the highest average TMB (13 mutations/MB) (4). A discrepancy between TMB between the primary and metastatic site was noticed in right-sided colon cancers (16.9\% vs. $6.9 \%$ ). Considering the poor disease control and rapid progression rates described aforementioned for 30\% of colorectal patients with MSI-H that receive immune checkpoint inhibitors, and the absence of predictive power of PD-L1 among patients with MSI-H CRC $(68,69)$, Schrock and collaborators assessed $\mathrm{TMB}$ as a predictor of response in this population. A total of 20 patient enrolled under the protocol received a PD-L1 inhibitor (19 pembrolizumab, 1 nivolumab). The authors found that patients with objective response had a median TMB of 54 mutations per Megabase; conversely non-responders had a median TMB of 29 mutations per Megabase (univariate analysis $\mathrm{P}<0.001$, in multivariate analysis $\mathrm{P}<0.1)$. The predictive power of TMB was also observed for disease control rate with non-responders having less than 37 and responders more than 41 mutations per Megabase (obtained by log-rank statistics) (70). Expanding to a large population database, the author mentions that $35 \%$ of patients lies below the TMB 37 mutations per Megabase cut-off, which coincides to the number of non-responder in this population. The caveat is that this cut-off may not apply for the combination nivolumab and ipilimumab in this setting (69). Besides, this is still a small population although retrospective studies based on current next generation sequencing and available clinical information can be extracted from the colorectal specimens from recent studies on the prevalence of immune checkpoint biomarkers (4), where only $17 \%$ of patients of 14 different gastrointestinal tumors who were MSI-H were also TMB-H.

As it was observed for PD-L1, PD-L2 also has a glycosylated isoform that serves a prognostic biomarker in CRC (71). Investigators aimed to evaluate glycosylation of PD-L2 in a detection sample of 124 patients with CRC later validated in an independent dataset of 232 patients (71). Strong PD-L2 expression correlated with INF- $\gamma$ mRNA expression, which is postulated to upregulate PD-L2 glycosylation. Poor survival correlated with strong PD-L2 in not only univariate (27.1 vs. 88.9 months; $\mathrm{P}=0.0002)$ but also multivariate analysis $(\mathrm{HR}=7.09$; 95\% CI, 1.78-28.16; $\mathrm{P}=0.005)$ in the validation cohort (71). Although the search for new checkpoints concurrently or sequenced 


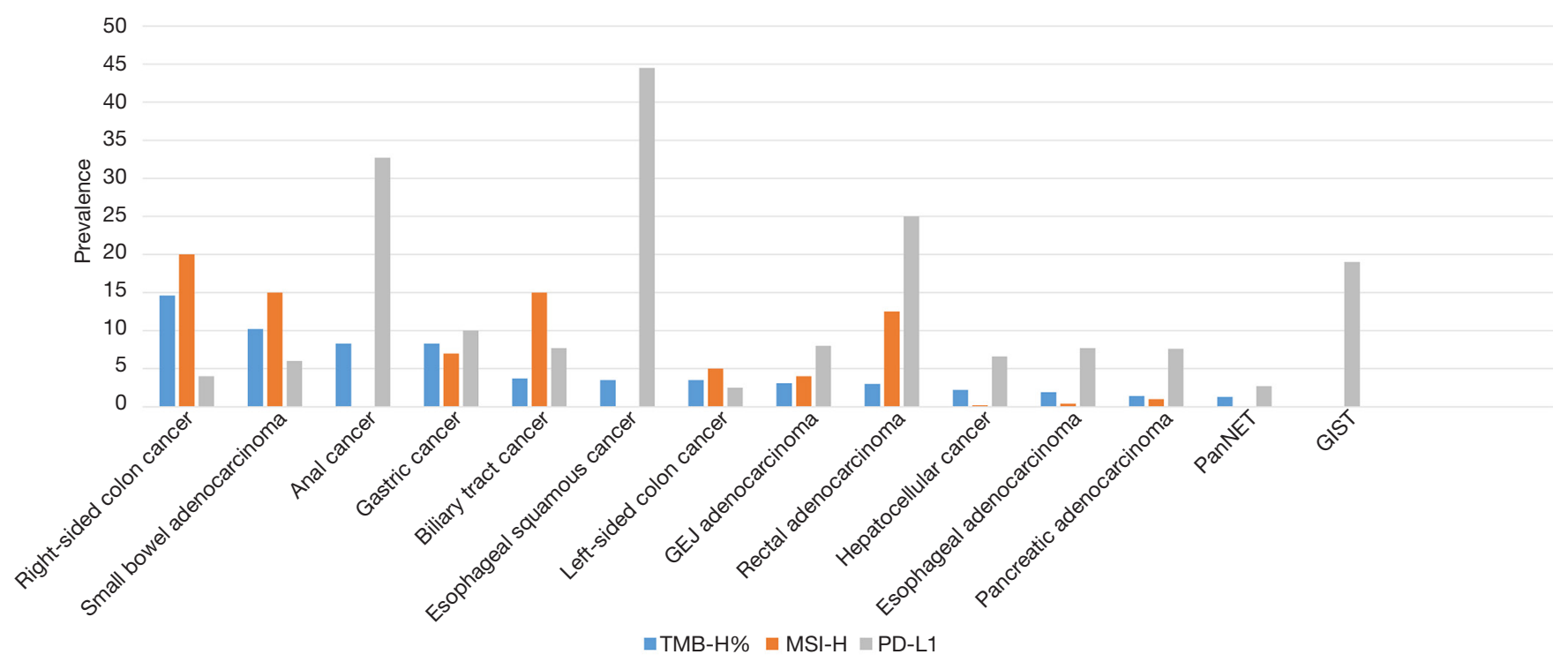

Figure 1 The prevalence of tumor mutational burden high (TMB-H), microsatellite instability high (MSI-H) and PD-L1 by gastrointestinal malignancy. Figures for biliary cancers for MSI are exclusively for extrahepatic biliary cancer. Large series evaluating MSI prevalence in gallbladder cancer document a less than 5\% prevalence. Ampullary carcinoma has a MSI prevalence of $10 \%$, while $3 \%$ of appendix carcinomas are considered MRD.

with the currently approved agents (LAG-3). In a study of mFOLFOX6, bevacizumab, durvalumab and the NKG2 NK cell checkpoint inhibitor monalizumab 17 patients were evaluable for response at 16 weeks resulting in a $53 \%$ partial responses $35 \%$ stable disease $12 \%$ progressive disease; there were no complete responses. Median time to response for the 7 confirmed responders was 15.4 weeks; median duration of response was not yet reached (72).

\section{Anal SCC}

It has been described that certain strains of the human papillomavirus (HPV) induce carcinogenesis by degrading p53 through its E6 protein and deleting Retinoblastoma $(\mathrm{Rb})$ protein by its E7 protein (73-75). By August 6th 2019 there is no FDA-approved ICB for anal SCC, nonetheless a cohort of patient with anal cancer with PD-L1 higher than $1 \%$ for a multi-histology (20 cancers) phase $1 \mathrm{~b}$ study showing a $17 \%$ ORR among 24 treated patient (86\% had prior therapy) and a $42 \%$ stable disease (SD) for a DCR of $59 \%$. The median OS of 9.3 months and mPFS of 3 months ranked as fair among the aforementioned trials $(76,77)$. NCI9673 was a phase 2 trial that enrolled 37 patients (HIV with CD $4<300 / \mathrm{mcL}$ allowed) in a single nivolumab arm after prior chemotherapy (median 2), achieving a 24\% ORR
< CR I 2 patients, but interestingly showing that baseline high TCD8 and Granzyme B, immune cell PD-L1 on IHC and TCD8 on flow cytometry were higher in responders than in non-responders and were not associated to sex, prior platinum, radiation or site of metastases (78). Currently, A multicenter study is randomizing patients with 6 virusassociated tumors (including HPV-positive anal SCC and EBV positive gastric cancer after preliminary results from gynecologic tumors) to nivolumab alone or in combination with CTLA-4, LAG3 or anti-CD38 monoclonal antibodies (79). Our center has enrolled patients to a combination of Listeria-based vaccine (presenting the HPV-16 E7 protein) and Intensity-Modulated Radiation Therapy (IMRT) associated with mytomycin and fluorouracil, showing safety, $80 \%$ complete response rate and $89 \%$ disease free survival at 34 months $(80,81)$.

Anal SCC have an intermediate rate of TMB-high (8.3\%). All TMB-high anal cancers were microsatellite stable (MSS). Although $80 \%$ of anal cancers are associated with HPV but only $8.3 \%$ are MSI-H, there may be other factors driving mutations, which can have clinical implications in view of the recent response rates of nivolumab in anal cancer MSI-H (78). Figure 1 shows the prevalence of MSI, PD-L1 and TMB-H in various gastrointestinal cancers. Table 1 shows an overview of 
Table 1 The landscape of clinical trials investigating immune checkpoint inhibitors in gastrointestinal malignancies with associated biomarkers when available Clinical outcomes are shown for each trial

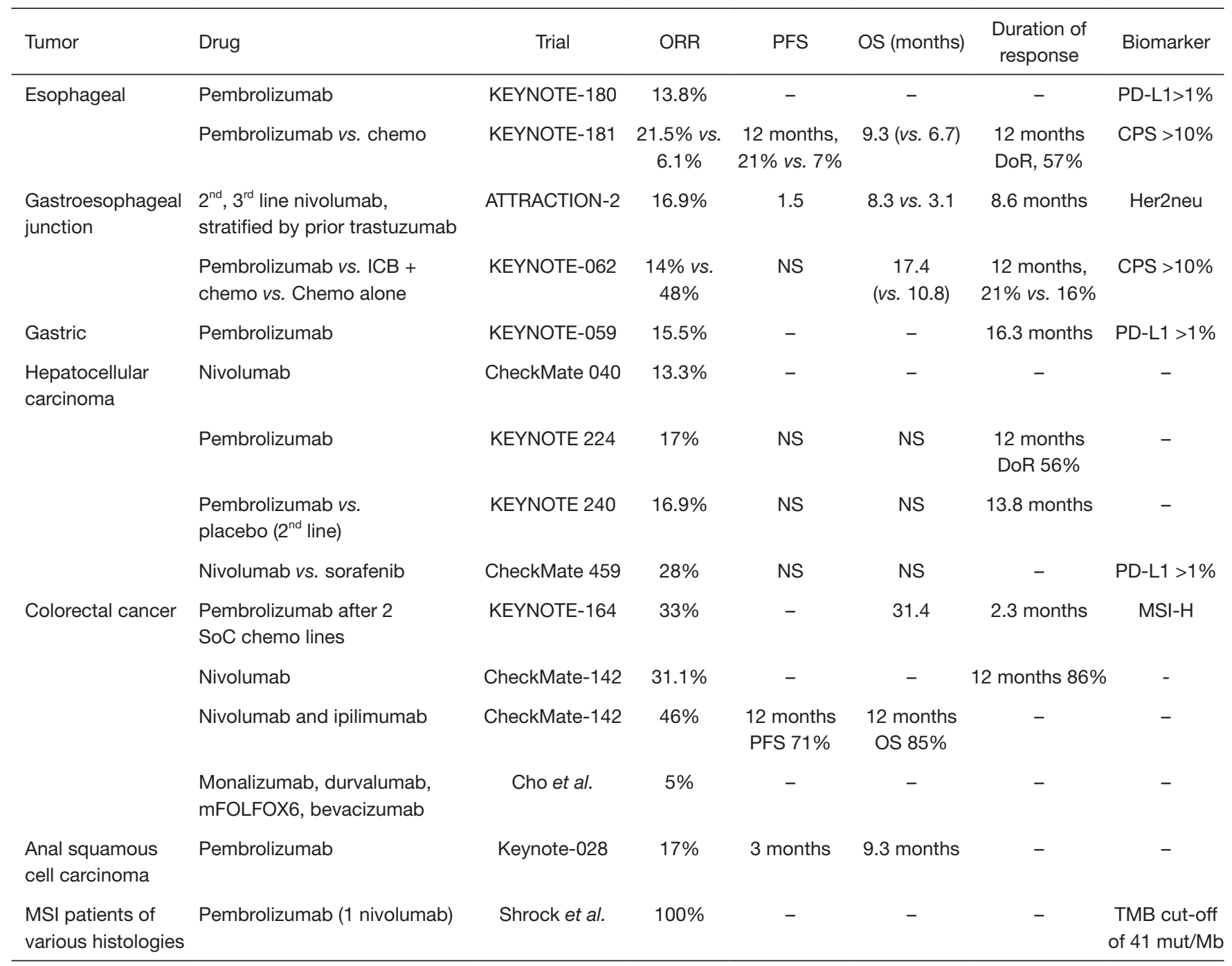

ORR, overall response rate; PFS, progression free survival; OS, overall survival; MSI, microsatellite instability; TMB, tumor mutational burden.

trials involving immune checkpoint inhibitors with their respective exploratory predictive biomarkers (and cut-offs).

\section{Prospective biomarkers}

\section{Gene alterations predicting acquired progression to immune checkpoint inhibitors}

A review this year of predictors of immune checkpoint inhibition at the European Society of Medical Oncology Meeting pinpointed the central role of upregulation of $\mathrm{PD}$ $\mathrm{L} 1$ in the tumor cell by interferon gama released by the
T CD8+ cell mounting an immune response (Figure 2). This process occurs after presentation of antigen by TCR and its co-receptor beta-2 microglobulin and is unleashed by binding of interferon gama to the IFNGR2 receptor, signaling by the JAK/STAT pathway and transcription of PD-L1 by the transcription factor interferon response factor 1 (IRF1). It is therefore not a surprise that gastric cancer has one the highest rates of mutations in antigen presentation pathways $(34.5 \%)$ and interferon-gama signaling pathway (20.4\%) (82). Investigators have also validated JAK2 loss of function mutations in patients receiving nivolumab and ipilimumab in the trial that lead to the agnostic approval of 


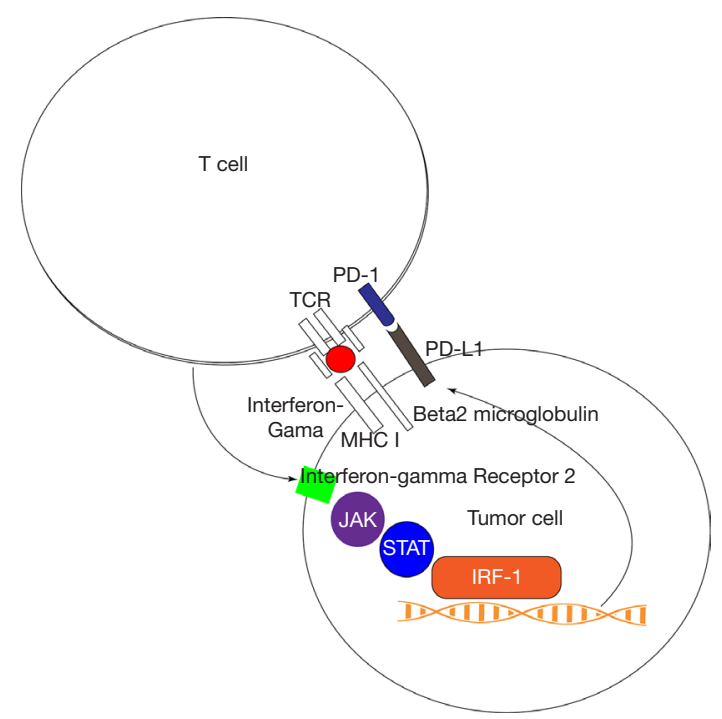

Figure 2 The activation of a T-cell by the presentation of an antigen (red sphere) by th Major Histocompatibility Complex (MHC) I and beta-microglobulin to the $\mathrm{T}$ cell receptor (TCR) promotes interferon gama release and stimulation in the tumor through interferon-gamma recetor 2 of PD-L1 through the JAKSTAT signaling pathway and its transcription factor (Interferonresponsive factor 1 (IRF-1). This diagram also illustrates why JAK and beta 2 microglobulin are potential biomarkers of immune checkpoint response.

nivolumab in solid tumors with MSI (83). In CRC the main proposed mechanisms of resistance to immune checkpoint inhibitors are reduced neoantigen generation, reduced MICA (the NKG2D ligand), induction of IDO (a promoter of T-cell exhaustion) and loss of function of IFN- $\gamma$ pathways (84-86). Interestingly, beta-2 microglobulin mutation or loss is not a negative predictor for response to immune checkpoints in MSI CRC (87). While New York University just launched a trial for combination of pembrolizumab with ruxolitinib in hematologic malignancies (NCT04016116), there is an ongoing combination trial of nivolumab and ruxolitinib in Hodgkin lymphoma (NCT03681561); the side effect profile and clinical outcomes from these trials will inform the solid tumor arena $(88,89)$.

\section{Gene aberrations predictive of hyper-progression}

Hyper-progression was defined as a more than twofold increase in tumor growth rate and was found in a study involving 113 to occurs in $9 \%$ of patients exposed to checkpoint inhibitors (90). That study showed hyper-progression in 2 patients with Gastrointestinal malignancies (colorectal, cholangiocarcinoma). Kato and collaborators described hyper-progression in all 6 patients with MDM4 or MDM6 amplifications and 2 out of 10 patients with EGFR among 155 patients (91). The possible mechanism through which immune checkpoint inhibitors causes hyper-progression is through upregulation of the interferon-related JAK/STAT pathway, with overexpression of interferon-regulatory factor 8 (IRF-8). Then, IRF-8 binds to the MDM2 promoter, increasing its transcription; this molecular event becomes significant in the $7 \%$ of cancers with MDM2 amplification). Kato also describes the prevalence of MDM2 amplifications in various tumors, including $11.2 \%$ in gallbladder adenocarcinomas, $7.84 \%$ in duodenal adenocarcinoma, $6.41 \%$ gastroesophagic cancer $5.5 \%$ in gastric adenocarcinoma and $5.24 \%$ in biliary tract cancer (92). To our knowledge, there is no publication on the prospective validation of these markers at the last cycles of chemotherapy to select patients to immune checkpoint inhibitors. By our own interrogation of publicly available gene datasets, MDM2 and MDM4 amplifications are seen in $5 \%$ of esophageal squamous, $6 \%$ of HCCs, $6 \%$ of biliary cancers, $5 \%$ of pancreatic cancers, $6.6 \%$ of CRCs (cBioportal based on TCGA Pan-Atlas gene Query). There is currently one phase 2 study investigating a MDM2 inhibitor (KRT-232) in patients with p53 wild type Merkel carcinomas who failed immune checkpoint inhibitors (NCT03787602). An ongoing study is recruiting patients with solid tumors for a combination of a MDM2 inhibitor (AlRN-692) with paclitaxel, which would be an interesting regimen for esophageal adenocarcinomas or Her2neu negative gastric adenocarcinoma with PD-L1 over $1 \%$ refractory or hyper-progressing on immune therapy (of note one patient with gastric cancer in the Kato study did not have hyper-progression). An active study aims to associate a MDM2 inhibitor with the MEK inhibitor trametinib in KRAS/BRAF mutated P53 wild type CRC patients (NCT03714958). Although there is no specified group of patients with MSI, this study will inform the safety of the drug and if viable for hyper-progression in CRC. There is a scarcity of case series of hyper-progression in gastrointestinal cancers. Finally, EGFR alterations activate the PD-L1 pathway, contributing to immune evasion (93). EGFR aberrations are observed in $4 \%$ of esophageal squamous, $2.3 \%$ of HCCs, $2.4 \%$ of biliary tract cancers, $4 \%$ of CRCs (cBioportal based on TCGA Pan-Atlas gene Query). 


\section{MSI and BRAF}

MSI is a negative predictive biomarker in metastatic colorectal adenocarcinoma with BRAF mutations. In a prospective population-based cohort study conducted in Scandinavia, 611 patients with colorectal adenocarcinoma had their tumor samples analyzed by immunohistochemistry for BRAF mutations and MSI. While $76 \%$ of MSI-H specimens concurrently carried mutated BRAF, $29 \%$ of mutated BRAF tumors were found to be MSI-H. The MSI-H in this cohort correlated with female age and age over 75 years old, right-sided tumors, mutated BRAF and lymph node metastases. Prolonged median progression free survival during first line chemotherapy was significantly associated with MSS tumors after multivariate analysis (HR 2.21; 95\% CI, 1.10-4.44; $\mathrm{P}=0.027$ ). Ten patients had concurrent MSI-H and mutated BRAF with a median overall survival of 1 month and median PFS (for those who received chemotherapy) of 2 months. The group concluded that the MSI is a poor predictor for patients with BRAF mutated colorectal adenocarcinoma receiving chemotherapy and immunotherapy should be investigated in the frontline setting (94). It would be interesting to evaluate clinical outcomes of patients with colorectal adenocarcinoma and BRAF mutations in the NCT02837263, which aims to associate pembrolizumab to SBRT in patients with CRC with isolated liver metastases. Of note, 7 out 84 patients with colorectal adenocarcinoma receiving combination atezolizumab and cobimetinib (only one of them MSI) had objective response (8\%), independent of their KRAS/BRAF status (95). Lastly, a case report has documented complete response in a patient with CRC metastatic to lymph nodes (biopsy-proven CRC metastasis to supraclavicular lymph node) and bones (for which the patient received radiation) with progression free survival at time of publication of 17 months (96).

\section{DNA polymerase germline mutations}

Three percent to $17 \%$ of CRCs occur before age 50 and are thus defined as early onset. Prior research has defined that germline mutations in the DNA Polymerase Epsilon (POLE) causes CRC (97) due to loss of its proofreading capability; this finding was associated to a hypermutated phenotype (98). Ahn et al. found 6 MSS non-polyposis syndrome early-onset CRC patients that had hypermutated tumors, 4 of these with a POLE P286R mutation. This mutation was validated in 83 MSS early-onset CRCs showing a prevalence of $7.2 \%$ in this subset of patients $(80 \%$ of them younger than 40 years old, $85 \%$ left-sided and $83 \%$ pure adenocarcinoma, $14 \%$ with mucinous feature). The TCGA study reported POLE mutation in 15 out of 224 cases (7\%). Of note, a second cohort of 27 patients with MSS late-onset CRC showed no POLE mutations, a potential biomarker for immunotherapy (99).

\section{The tumor microenvironment}

Tauriello et al. developed an experimental mice model of MSS, low mutation burden colorectal metastasis with $\mathrm{T}$ cell exclusion due to a TGF-beta induced abundant desmoplastic reaction refractory to immune checkpoint blockade. Subsequently, the group showed that treatment with the TGF-beta inhibitor galunisertib rendered liver metastases susceptive to PD-1 inhibition (100). To exemplify the search for a TGF-beta biomarker, the combination of galunisertib and sorafenib has showed a $64 \%$ disease control rate and median overall survival of 18 months and a good safety profile in patients with HCC. Responders, defined as $20 \%$ change in circulating TGF-Beta 1 , had a median overall survival of 22.8 months as opposed to non-responders, who had a median overall survival of 12 months (101). Currently Galunisertib has been investigated with durvalumab in pancreatic cancer (NCT02734160) and with nivolumab in HCC (NCT02423343). There are no trial open for CRC with liver metastasis.

\section{The human microbiome and GI cancers}

Retrospective data from 2 Londonian centers encompassing 196 patients (mostly non-small cell lung cancer and melanoma patients) have shown that antibiotics administered within 1 month of immune checkpoint inhibitors may dampen the overall response (refractoriness increased from $44 \%$ to $81 \%, \mathrm{P}<0.001$ ) and survival ( 2 vs. 26 months, $\mathrm{P}<0.001)$ rates derived from these drugs. Among the patients, $35 \%$ received antibiotics concurrently and $15 \%$ antibiotics within 1 months of checkpoint inhibitor infusions. The results were presented at the 2019 ASCOsociety for immunotherapy of cancer (SITC) Clinical Immuno-Oncology Symposium corroborating prior data on correlation of gut microbiome with $\mathrm{PD}-1$ inhibition response (102-104). However, there was no detrimental effect with concurrent antibiotic and immunotherapy. NCT02960282 plan to enroll 80 patients with metastatic CRC randomized to three first line arms: immune 
checkpoint, FOLFIRI or FOLFOX, with baseline and interval collection of stools to correlate response to specific gut microbiome species.

\section{RNA-editing}

APOBEC (Apolipoprotein B mRNA editing enzyme, catalytic peptide-like) isoforms converts cytosine to uracyl as a posttranscriptional modification of the messenger RNA molecule but is also responsible for $\mathrm{C}-\mathrm{T}$ genomic conversion events that begets an APOBEC genomic signature (105-111). Recently, Wang et al. have shown that the mutational signature of APOBEC $3 \mathrm{~B}$ is a better biomarker for durable clinical response to immunotherapy in nonsmall cell lung cancer than total mutation count (105). APOBEC over-activity was initially described in gastric and CRCs in 2013, based on the sequencing of 2,680 tumors from The Cancer Genome Atlas (106).

APOBEC 1 defines a distinct mutational signature in esophageal carcinoma $(112,113)$. Cytotoxin-associated gene A (CagA) decreases expression of APOBEC $3 \mathrm{~A}$, $\mathrm{APOBEC} 3 \mathrm{C}$ and $\mathrm{APOBEC} 3 \mathrm{~F}$ in gastric cancer and is linked to Cag+ Helicobacter pylori strains (114). Furthermore, the overexpression of the developmental protein NKX6.3 downregulates APOBEC in gastric cancer and APOBEC seems to be therefore downregulated in subsets of patients with gastric cancer (115). In pancreatic ductal adenocarcinoma (PDA), APOBEC overexpression is associated with significantly decreased survival in early stage patients (116), and the APOBEC3A isoform is associated with CD8+ $\mathrm{T}$ exhaustion and $\mathrm{T}$ and $\mathrm{B}$ cells number and distribution (117).

An abstracted published in the proceedings but not presented at the American Association of Clinical research in 2015 investigated the related the role of microRNA-122, DNA methylation and APOBEC 1 and 3 overexpression to the carcinogenesis of HCC (118). APOBEC3 was correlated with high levels of T CD8+ lymphocyte cytotoxic response (as assessed by granzyme and perforin levels in pre-clinical models) in HCC (119). The APOBEC was linked to a subset of colorectal carcinomas with CXCR4/CREB pathway overactivity (120) and a spliced isoform of APOBEC1 is overexpressed in human colon cancer (121). As a proportion of BRAF-V600 mutant melanomas fail BRAF inhibitor treatment (vemurafenib or dabrafenib) due to cytosine mutations in MEK1, MEK2, or other signal transduction pathway genes potentially mediated by APOBEC3 deamination, it would be interesting to know if this is a relevant mechanism of resistance of BRAF V600 mutant colon cancer failing irinotecan, cetuximab and vemurafenib, which is in advanced clinical trial development (122). Finally, chromosomal breakage due to DNA repeats was linked to an APOBEC signature in breast cancer and no pre-clinical studies in CRC, where a defined CIMP subset is characterized by high level of aneuploidy, have been developed.

\section{Conclusions}

TMB-high was associated with MSI-High. Among MSS tumors, squamous cell cancers had the highest TMB-high rate $(8.3 \%$ for anal and $3.5 \%$ for esophageal primaries), while pancreatic neuroendocrine and gastrointestinal stromal tumors had the lowest rate (1.3\% and $0 \%$ respectively).

The landscape of predictive biomarkers for immune checkpoint inhibitors in gastrointestinal cancers continues to expand. The initial focus was on neoantigen formation, checkpoints, cytokines and immune genes signatures on T-cells and tumors. It now includes the desmoplastic reaction players such as tumor infiltrative lymphocytes and density, Myeloid-derived Suppressor cells (MDSC), and M2 macrophages. New processes of neo-antigen formation such as APOBEC homologous repair deficiency and DNA polymerase mutations may gain momentum in this setting. At last, careful drug development will run biomarkers of response in parallel to predictors of hyper-progression, forecast mechanisms of acquired resistance and heat the cold immune environment of gastrointestinal tumors.

\section{Acknowledgments}

None.

\section{Footnote}

Conflicts of Interest: The authors have no conflicts of interest to declare.

Ethical Statement: The authors are accountable for all aspects of the work in ensuring that questions related to the accuracy or integrity of any part of the work are appropriately investigated and resolved.

\section{References}

1. Bartley ANN, Luthra R, Saraiva DS, et al. Identification of 
cancer patients with Lynch syndrome: clinically significant discordances and problems in tissue-based mismatch repair testing. Cancer Prev Res (Phila) 2012;5:320-7.

2. Cicek MS, Lindor NM, Gallinger S, et al. Quality assessment and correlation of microsatellite instability and immunohistochemical markers among populationand clinic-based colorectal tumor results from the Colon Cancer Family Registry. J Mol Diagn 2011;13:271-81.

3. Lee JH, Cragun D, Thompson Z, et al. Association between IHC and MSI testing to identify mismatch repairdeficient patients with ovarian cancer, Genet Test Mol Biomarkers 2014;18:229-35.

4. Salem ME, Puccini A, Grothey A, et al. Landscape of Tumor Mutation Load, Mismatch Repair Deficiency, and PD-L1 Expression in a Large Patient Cohort of Gastrointestinal Cancers. Mol Cancer Res 2018;16:805-12.

5. Hall MJ, Gowen K, Sanford EM, et al. Evaluation of microsatellite instability status and gastrointestinal tumor samples tested with comprehensive genomic profiling. J Clin Oncol 2016;34:528.

6. Kim H, An J, Noh S, et al. High microsatellite instability predicts good prognosis in intestinal-type gastric cancers. J Gastroenterol Hepatol 2011;26:585-92.

7. Corso G, Pedrazzani C, Marrelli D, et al. Correlation of microsatellite instability at multiple loci with longterm survival in advanced gastric carcinoma. Arch Surg 2009;144:722-7.

8. Beghelli S, de Manzoni G, Barbi S, et al. Microsatellite instability in gastric cancer is associated with better prognosis in only stage II cancers. Surgery 2006;139:347-56.

9. Choi SW, Choi JR, Chung YJ, et al. Prognostic implications of microsatellite genotypes in gastric carcinoma. Int J Cancer 2000;89:378-83.

10. Falchetti M, Saieva C, Lupi R, et al. Gastric cancer with high-level microsatellite instability: Target gene mutations, clinicopathologic features, and long-term survival. Hum Pathol 2008;39:925-32.

11. Oliveira C, Seruca R, Seixas M, et al. The clinicopathological features of gastric carcinomas with microsatellite instability may be mediated by mutations of different 'target genes': A study of the TGFbeta RII, IGFII R, and BAX genes. Am J Pathol 1998;153:1211-9.

12. Schneider BG, Bravo JC, Roa JC, et al. Microsatellite instability, prognosis and metastasis in gastric cancers from a low-risk population. Int J Cancer 2000;89:444-52.

13. Yamamoto H, Perez Piteira J, Yoshida T, et al. Gastric cancers of the microsatellite mutator phenotype display characteristic genetic and clinical features.
Gastroenterology 1999;116:1348-57.

14. Seo HM, Chang Y, Joo S, et al. Clinicopathologic characteristics and outcomes of gastric cancers with the MSI-H phenotype. J Surg Oncol 2009;99:143-7.

15. An JY, Kim H, Cheong J, et al. Microsatellite instability in sporadic gastric cancer: Its prognostic role and guidance for 5 -FU based chemotherapy after R0 resection. Int J Cancer 2012;131:505-11.

16. Kim JY, Shin N, Kim A, et al. Microsatellite instability status in gastric cancer: A reappraisal of its clinical significance and relationship with mucin phenotypes. Korean J Pathol 2013;47:28-35.

17. Oki E, Kakeji Y, Zhao Y, et al. Chemosensitivity and survival in gastric cancer patients with microsatellite instability. Ann Surg Oncol 2009;16:2510-5.

18. Bacani J, Zwingerman R, Di Nicola N, et al. Tumor microsatellite instability in early onset gastric cancer. J Mol Diagn 2005;7:465-77.

19. Hsieh CC, Hsu HS, Li AF, et al. Clinical relevance of PDL1 and PD-L2 overexpression in patients with esophageal squamous cell carcinoma. J Thorac Dis 2018;10:4433-44.

20. Dmitrienko A, D'Agostino RB Sr. Multiplicity Considerations in Clinical Trials. N Engl J Med 2018;378:2115-22.

21. Tabernero J, Cutsem EV, Bang YJ, et al. Pembrolizumab with or without chemotherapy versus chemotherapy for advanced gastric or gastroesophageal junction (G/GEJ) adenocarcinoma: The phase III KEYNOTE-062 study. J Clin Oncol 2019;37:abstr LBA4007.

22. Derks S, Nason KS, Liao X, et al. Epithelial PD-L2 Expression Marks Barrett's Esophagus and Esophageal Adenocarcinoma. Cancer Immunol Res 2015;3:1123-9.

23. Fuchs CS, Doi T, Jang RW, et al. Safety and Efficacy of Pembrolizumab Monotherapy in Patients With Previously Treated Advanced Gastric and Gastroesophageal Junction Cancer: Phase 2 Clinical KEYNOTE-059 Trial. JAMA Oncol 2018;4:e180013.

24. Bang YJ, Kang YK, Catenacci DV, et al. Pembrolizumab alone or in combination with chemotherapy as firstline therapy for patients with advanced gastric or gastroesophageal junction adenocarcinoma: results from the phase II nonrandomized KEYNOTE-059 study. Gastric Cancer 2019;22:828-37.

25. Shah MA, Kojima T, Hochhauser D, et al. Efficacy and Safety of Pembrolizumab for Heavily Pretreated Patients With Advanced, Metastatic Adenocarcinoma or Squamous Cell Carcinoma of the Esophagus: The Phase 2 KEYNOTE-180 Study. JAMA Oncol 2019;5:546-50. 
26. Kojima T, Muro K, Francois E, et al. Pembrolizumab versus chemotherapy as second-line therapy for advanced esophageal cancer: The phase 3 KEYNOTE-181 study. J Clin Oncol 2019;37:2.

27. Goggins M, Offerhaus GJ, Hilgers W, et al. Pancreatic adenocarcinomas with DNA replication errors (RER) are associated with wild-type K-ras and characteristic histopathology. poor differentiation, a syncytial growth pattern, and pushing borders suggest RER. Am J Pathol 1998;152:1501-7.

28. Nakata B, Wang Y, Yashiro M, et al. Negative hMSH2 protein expression in pancreatic carcinoma may predict a better prognosis of patients. Oncol Rep 2003;10:997-1000.

29. Yamamoto H, Itoh F, Nakamura H, et al. Genetic and clinical features of human pancreatic ductal adenocarcinomas with widespread microsatellite instability. Cancer Res 2001;61:3139-44.

30. Royal RE, Levy C, Turner K, et al. Phase 2 trial of single agent Ipilimumab (anti-CTLA-4) for locally advanced or metastatic pancreatic adenocarcinoma. J Immunother 2010;33:828-33.

31. Brahmer JR, Tykodi SS, Chow LQ, et al. Safety and activity of anti-PD-L1 antibody in patients with advanced cancer. N Engl J Med 2012;366:2455-65.

32. Herbst RS, Soria JC, Kowanetz M, et al. Predictive correlates of response to the anti-PD-L1 antibody MPDL3280A in cancer patients. Nature 2014;515:563-7.

33. Melisi D, Garcia-Carbonero R, Macarulla T, et al. A phase II, double-blind study of galunisertib+gemcitabine (GG) vs gemcitabine+placebo (GP) in patients (pts) with unresectable pancreatic cancer (PC). J Clin Oncol 2016;34:4019.

34. Oettle H, Seufferlein T, Luger T, et al. Final results of a phase I/II study in patients with pancreatic cancer, malignant melanoma, and colorectal carcinoma with trabedersen. J Clin Oncol 2012;30:4034.

35. Hecht JR, Bedford R, Abbruzzese JL, et al. A phase I/II trial of intratumoral endoscopic ultrasound injection of ONYX-015 with intravenous gemcitabine in unresectable pancreatic carcinoma. Clin Cancer Res 2003;9:555-61.

36. Stromnes IM, Schmitt TM, Hulbert A, et al. T Cells Engineered against a Native Antigen Can Surmount Immunologic and Physical Barriers to Treat Pancreatic Ductal Adenocarcinoma. Cancer Cell 2015;28:638-52.

37. Beatty GL, Haas AR, Maus MV, et al. Mesothelin-specific chimeric antigen receptor mRNA-engineered $\mathrm{T}$ cells induce anti-tumor activity in solid malignancies. Cancer Immunol Res 2014;2:112-20.

38. Beatty GL, O'Hara MH, Lacey SF, et al. Activity of mesothelin-specific chimeric antigen receptor $\mathrm{T}$ cells against pancreatic carcinoma metastases in a phase 1 trial. Gastroenterology 2018;155:29-32.

39. Thistlethwaite FC, Gilham DE, Guest RD, et al. The clinical efficacy of first-generation carcinoembryonic antigen (CEACAM5)-specific CAR T cells is limited by poor persistence and transient pre-conditioning-dependent respiratory toxicity. Cancer Immunol Immunother 2017;66:1425-36.

40. Ang C, Klempner SJ, Ali SM, et al. Prevalence of established and emerging biomarkers of immune checkpoint inhibitor response in advanced hepatocellular carcinoma. Oncotarget 2019;10:4018-25.

41. El-Khoueiry AB, Sangro B, Yau T, et al. Nivolumab in patients with advanced hepatocellular carcinoma (CheckMate 040): an open-label, non-comparative, phase $1 / 2$ dose escalation and expansion trial. Lancet 2017;389:2492-502.

42. Zhu AX, Finn RS, Edeline J et al. Pembrolizumab in patients with advanced hepatocellular carcinoma previously treated with sorafenib (KEYNOTE-224): a non-randomised, open-label phase 2 trial. Lancet Oncol 2018;19:940-52.

43. Finn RS, Ryoo BY, Merle P, et al. Results of KEYNOTE-240: phase 3 study of pembrolizumab (Pembro) vs best supportive care (BSC) for second line therapy in advanced hepatocellular carcinoma (HCC). J Clin Oncol 2019;37:4004.

44. Sangro B, Park JW, Dela Cruz CM, et al. A randomized, multicenter, phase 3 study of nivolumab vs sorafenib as first-line treatment in patients (pts) with advanced hepatocellular carcinoma (HCC): CheckMate-459. J Clin Oncol 2013;34:TPS4147.

45. Goodman AM, Kato S, Bazhenova L, et al. Tumor Mutational Burden as an Independent Predictor of Response to Immunotherapy in Diverse Cancers. Mol Cancer Ther 2017;16:2598-608.

46. Yang H, Zhou X, Sun L, et al. Correlation Between PDL2 Expression and Clinical Outcome in Solid Cancer Patients: A Meta-Analysis. Front Oncol 2019;9:47.

47. Roa JC, Roa I, Correa P, et al. Microsatellite instability in preneoplastic and neoplastic lesions of the gallbladder. J Gastroenterol 2005;40:79-86.

48. Suto T, Habano W, Sugai T, et al. Infrequent microsatellite instability in biliary tract cancer. J Surg Oncol 2001;76:121-6.

49. Ruemmele P, Dietmaier W, Terracciano L, et al. Histopathologic features and microsatellite instability of 
cancers of the papilla of vater and their precursor lesions. Am J Surg Pathol 2009;33:691-704.

50. Kim MH, Jang M, Kim H et al. Distinct immunological properties of the two histological subtypes of adenocarcinoma of the ampulla of Vater. Cancer Immunol Immunother 2019;68:443-54.

51. Brueckl WM, Heinze E, Milsmann C, et al. Prognostic significance of microsatellite instability in curatively resected adenocarcinoma of the small intestine. Cancer Lett 2004;203:181-90.

52. Achille A, Baron A, Zamboni G, et al. Molecular pathogenesis of sporadic duodenal cancer. Br J Cancer 1998;77:760-5.

53. Potter DD, Murray J, Donohue J, et al. The role of defective mismatch repair in small bowel adenocarcinoma in celiac disease. Cancer Res 2004;64:7073-7.

54. Cunningham JM, Kim CY, Christensen ER, et al. The frequency of hereditary defective mismatch repair in a prospective series of unselected colorectal carcinomas. Am J Hum Genet 2001;69:780-90.

55. Vilar E, Gruber SB. Microsatellite instability in colorectal cancer-the stable evidence. Nat Rev Clin Oncol 2010;7:153-62.

56. Iacopetta B, Grieu F, Amanuel B. Microsatellite instability in colorectal cancer. Asia Pac J Clin Oncol 2010;6:260-9.

57. Iachetta F, Domati F, Reggiani-Bonetti L, et al. Prognostic relevance of microsatellite instability in pT3N0M0 colon cancer: a population-based study. Intern Emerg Med 2016;11:41-6.

58. Taieb J, Shi Q, Pederson L, et al. Prognosis of microsatellite instability and/or mismatch repair deficiency stage III colon cancer patients after disease recurrence following adjuvant treatment: results of an ACCENT pooled analysis of seven studies. Ann Oncol 2019;30:1466-71.

59. Kim ST, Lee J, Park SH, et al. The effect of DNA mismatch repair (MMR) status on oxaliplatin-based firstline chemotherapy as in recurrent or metastatic colon cancer. Med Oncol 2010;27:1277-85.

60. Koopman M, Kortman GA, Mekenkamp L, et al. Deficient mismatch repair system in patients with sporadic advanced colorectal cancer. Br J Cancer 2009;100:266-73.

61. Taggart MW, Galbincea J, Mansfield PF, et al. High-level microsatellite instability in appendiceal carcinomas. Am J Surg Pathol 2013;37:1192-200.

62. Karahan B, Argon A, Y1ldırım M, et al. Relationship between MLH-1, MSH-2, PMS-2, MSH-6 expression and clinicopathological features in colorectal cancer. Int J Clin
Exp Pathol 2015;8:4044-53.

63. Belt EJ, te Velde EA, Krijgsman O, et al. High lymph node yield is related to microsatellite instability in colon cancer. Ann Surg Oncol 2012;19:1222-30.

64. Sargent DJ, Marsoni S, Monges G, et al. Defective mismatch repair as a predictive marker for lack of efficacy of fluorouracil-based adjuvant therapy in colon cancer. J Clin Oncol 2010;28:3219-26.

65. Kim ST, Lee J, Park SH, et al. Clinical impact of microsatellite instability in colon cancer following adjuvant FOLFOX therapy. Cancer Chemother Pharmacol 2010;66:659-67.

66. Lim YJ, Koh J, Kim S, et al. Chemoradiation-Induced Alteration of Programmed Death-Ligand 1 and CD8+ Tumor-Infiltrating Lymphocytes Identified Patients With Poor Prognosis in Rectal Cancer: A Matched Comparison Analysis. Int J Radiat Oncol Biol Phys 2017;99:1216-24.

67. Le D, Kavan P, Kim T, et al. Safety and antitumor activity of pembrolizumab in patients with advanced microsatellite instability-high (MSI-H) colorectal cancer: KEYNOTE-164. Ann Oncol 2018;29:mdy149.020.

68. Overman MJ, McDermott R, Leach JL, et al. Nivolumab in patients with metastatic DNA mismatch repair-deficient or microsatellite instability-high colorectal cancer (CheckMate 142): an open-label, multicentre, phase 2 study. Lancet Oncol 2017;18:1182-91.

69. Overman MJ, Lonardi S, Wong KYM, et al. Durable Clinical Benefit With Nivolumab Plus Ipilimumab in DNA Mismatch Repair-Deficient/Microsatellite Instability-High Metastatic Colorectal Cancer. J Clin Oncol 2018;36:773-9.

70. Schrock AB, Ouyang C, Sandhu J, et al. Tumor mutational burden is predictive of response to immune checkpoint inhibitors in MSI-high metastatic colorectal cancer. Ann Oncol 2019;30:1096-103.

71. Wang H, Yao H, Li C, et al. PD-L2 expression in colorectal cancer: Independent prognostic effect and targetability by deglycosylation. Oncoimmunology 2017;6:e1327494.

72. Cho M, Bendell JC, Han S, et al. Durvalumab, monalizumab, mFOLFOX6, and bevacizumab in patients with metastatic microsatellite-stable colorectal cancer. In: ESMO annual meeting 2019. Ann Oncol 2019;30:v475-532.

73. Werness BA, Levine AJ, Howley PM. Association of human papillomavirus types 16 and 18 E6 proteins with p53. Science 1990;248:76-9.

74. Li X, Coffino P. High-risk human papillomavirus E6 protein has two distinct binding sites within $\mathrm{p} 53$, of which only one determines degradation. J Virol 1996;70:4509-16.

75. Balsitis SJ, Sage J, Duensing S, et al. Recapitulation of the 
effects of the human papillomavirus type $16 \mathrm{E} 7$ oncogene on mouse epithelium by somatic Rb deletion and detection of $\mathrm{pRb}$-independent effects of $\mathrm{E} 7$ in vivo. Mol Cell Biol 2003;23:9094-103.

76. Ott PA, Bang YJ, Piha-Paul SA. T-cell-inflamed geneexpression profile, programmed death ligand 1 expression, and tumor mutational burden predict efficacy in patients treated with pembrolizumab across 20 cancers: KEYNOTE-028. J Clin Oncol 2019;37:318-27.

77. Ott PA, Piha-Paul SA, Munster P, et al. Safety and antitumor activity of the anti-PD-1 antibody pembrolizumab in patients with recurrent carcinoma of the anal canal. Ann Oncol 2017;28:1036-41.

78. Morris VK, Salem ME, Nimeiri H, et al. Nivolumab for previously treated unresectable metastatic anal cancer (NCI9673): a multicentre, single-arm, phase 2 study. Lancet Oncol 2017;18:446-53.

79. Hollebecque A, Meyer T, Moore KN, et al. An open-label, multicohort, phase I/II study of nivolumab in patients with virus-associated tumors (CheckMate 358): Efficacy and safety in recurrent or metastatic (R/M) cervical, vaginal, and vulvar cancers. J Clin Oncol 2017;35:5504.

80. Safran H, Leonard KL, Perez K, et al. Tolerability of ADXS11-001 Lm-LLO Listeria-Based Immunotherapy With Mitomycin, Fluorouracil, and Radiation for Anal Cancer. Int J Radiat Oncol Biol Phys 2018;100:1175-8.

81. Safran H, Leonard KL, DiPetrillo TA, et al. ADXS11-001 Lm-LLO Immunotherapy, Mitomycin, 5-fluorouracil (5FU) and Intensity-modulated radiation therapy (IMRT) for Anal Cancer. J Clin Oncol 2017;35:e15072.

82. Budczies J, Bockmayr M, Klauschen F, et al. Mutation patterns in genes encoding interferon signaling and antigen presentation: A pan-cancer survey with implications for the use of immune checkpoint inhibitors. Genes Chromosomes Cancer 2017;56:651-9.

83. Kopetz S, Andre T, Overman MJ, et al. Exploratory analysis of Janus kinase 1 (JAK1) loss-of-function (LoF) mutations in patients with DNA mismatch repairdeficient/microsatellite instability-high (dMMR/MSI-H) metastatic colorectal cancer $(\mathrm{mCRC})$ treated with nivolumab + ipilimumab in CheckMate-142. In: AACR Annual Meeting 2018. Cancer Res 2018;78:Abstract 2603.

84. Rooney MS, Shukla SA, Wu CJ, et al. Molecular and genetic properties of tumors associated with local immune cytolytic activity. Cell 2015;160:48-61.

85. Salih HR, Rammensee HG, Steinle A. Cutting edge: Down-regulation of MICA on human tumors by proteolytic shedding. J Immunol 2002;169:4098-102.
86. Moon YW, Hajjar J, Hwu P, et al. Targeting the indoleamine 2,3-dioxygenase pathway in cancer. J Immunother Cancer 2015;3:51.

87. Middha S, Yaeger R, Shia J, et al. Majority of B2M-Mutant and -Deficient Colorectal Carcinomas Achieve Clinical Benefit From Immune Checkpoint Inhibitor Therapy and Are Microsatellite Instability-High. JCO Precis Oncol 2019;3. doi: 10.1200/PO.18.00321.

88. Bu X, Mahoney KM, Freeman GJ. Learning from PD-1 resistance: new combination strategies. Trends Mol Med 2016;22:448-51.

89. Horn S, Leonardelli S, Sucker A, et al. Tumor CDKN2AAssociated JAK2 Loss and Susceptibility to Immunotherapy Resistance. J Natl Cancer Inst 2018;110:677-81

90. Champiat S, Dercle L, Ammari S, et al. Hyper-progressive disease is a new pattern of progression in cancer patients treated by anti-PD-1/PD-L1. Clin Cancer Res 2017;23:1920-8.

91. Kato S, Goodman A, Walavalkar V. Hyperprogressors after Immunotherapy: Analysis of Genomic Alterations Associated with Accelerated Growth Rate. Clin Cancer Res 2017;23:4242-50.

92. Kato S, Ross JS, Gay L, et al. Analysis of MDM2 amplification: next-generation sequencing of patients with diverse malignancies. JCO Precis Oncol 2018;2018. doi: 10.1200/PO.17.00235.

93. Akbay EA, Koyama S, Carretero J, et al. Activation of the PD-1 pathway contributes to immune escape in EGFRdriven lung tumors. Cancer Discov 2013;3:1355-63.

94. Aasebø KØ, Dragomir A, Sundström M, et al. Consequences of a high incidence of microsatellite instability (non-Lynch) and BRAF mutated tumors in a population based cohort of metastatic colorectal cancer. Cancer Med 2019;8:3623-35.

95. Hellmann MD, Kim TW, Lee CB, et al. Phase Ib study of atezolizumab combined with cobimetinib in patients with solid tumors. Ann Oncol 2019;30:1134-42.

96. Sehdev A, Cramer HM, Ibrahim AA, et al. Pathological complete response with anti-PD-1 therapy in a patient with microsatellite instable high, BRAF mutant metastatic colon cancer: a case report and review of literature. Discov Med 2016;21:341-7.

97. Palles C, Cazier JB, Howarth KM, et al. Germline mutations affecting the proofreading domains of POLE and POLD1 predispose to colorectal adenomas and carcinomas. Nat Genet 2013;45:136-44.

98. Kane DP, Shcherbakova PV. A common cancer-associated DNA polymerase $\varepsilon$ mutation causes an exceptionally 
strong mutator phenotype, indicating fidelity defects distinct from loss of proofreading. Cancer Res 2014;74:1895-901.

99. Ahn SM, Ansari AA, Kim J. The somatic POLE P286R mutation defines a unique subclass of colorectal cancer featuring hypermutation, representing a potential genomic biomarker for immunotherapy. Oncotarget 2016;7:68638-49.

100. Tauriello DVF, Palomo-Ponce S, Stork D, et al. TGF $\beta$ drives immune evasion in genetically reconstituted colon cancer metastasis. Nature 2018;554:538-43.

101. Kelley RK, Gane E, Assenat E, et al. A Phase 2 Study of Galunisertib (TGF- $\beta 1$ Receptor Type I Inhibitor) and Sorafenib in Patients With Advanced Hepatocellular Carcinoma. Clin Transl Gastroenterol 2019;10:e00056.

102. Routy B, Le Chatelier E, Derosa L, et al. Gut microbiome influences efficacy of PD-1-based immunotherapy against epithelial tumors. Science 2018;359:91-7.

103. Bhatt AP, Redinbo MR, Bultman SJ. The role of the microbiome in cancer development and therapy. CA Cancer J Clin 2017;67:326-44.

104. Sivan A, Corrales L, Hubert N, et al. Commensal Bifidobacterium promotes antitumor immunity and facilitates anti-PD-L1 efficacy. Science 2015;350:1084-9.

105. Wang S, Jia M, He Z, et al. APOBEC3B and APOBEC mutational signature as potential predictive markers for immunotherapy response in non-small cell lung cancer. Oncogene 2018;37:3924-36.

106. Roberts SA, Lawrence MS, Klimczak LJ, et al. An APOBEC cytidine deaminase mutagenesis pattern is widespread in human cancers. Nat Genet 2013;45:970-6.

107. Burns MB, Temiz NA, Harris RS, et al. Evidence for APOBEC3B mutagenesis in multiple human cancers. Nat Genet 2013;45:977-83.

108.Jia P, Pao W, Zhao Z, et al. Patterns and processes of somatic mutations in nine major cancers. BMC Med Genomics 2014;7:11.

109. Roberts SA, Gordenin DA. Hypermutation in human cancer genomes: footprints and mechanisms. Nat Rev Cancer 2014;14:786-800.

110. Witkin KL, Hanlon SE, Strasburger JA, et al. RNA editing, epitranscriptomics, and processing in cancer progression. Cancer Biol Ther 2015;16:21-7.

111. Gansmo LB, Romundstad P, Hveem K, et al. APOBEC3A/ $\mathrm{B}$ deletion polymorphism and cancer risk carcinogenesis. Carcinogenesis 2018;39:118-24.

112. Saraconi G, Severi F, Sala C, et al. The RNA editing enzyme APOBEC1 induces somatic mutations and a compatible mutational signature is present in esophageal adenocarcinomas. Genome Biol 2014;15:417.

113.Zhang L, Zhou Y, Cheng C, et al. Genomic analyses reveal mutational signatures and frequently altered genes in esophageal squamous cell carcinoma. Am J Hum Genet 2015;96:597-611.

114. Yoon JH, Kim O, Eun JW, et al. Multiple genetic mutations caused by NKX6.3 depletion contribute to gastric tumorigenesis. Sci Rep 2018;8:17609.

115.Zhang B, Zhang X, Jin M, et al. CagA increases DNA methylation and decreases PTEN expression in human gastric cancer. Mol Med Rep 2019;19:309-19.

116. Woermann S, Cowan R, Ross SM, et al. APOBEC3A inhibits tumor immune response independent of deamination in a novel genetic model of pancreatic cancer. Cancer Res 2018;78:4751.

117. Tsou AP, Wang KH, Huang HY, et al. MicroRNA-122 is a potential regulator of DNA methylation partly by affecting the global level profile of 5hmC. Cancer Res 2015;75:176.

118. Kawaguchi T, Yan L, Takahashi H, et al. Intra-tumor immune activity is linked to genetic diversity of tumor infiltrating lymphocyte and impact clinical outcomes in hepatocellular cancer. Proceedings of the 110th Annual Meeting of the American Association for Cancer Research; 2019 Mar 29-Apr 3; Atlanta, GA. Philadelphia (PA); AACR; 2019. Abstract nr 4562.

119. Katkoori V, Anderson Z, Manne U, et al. Inhibition of CXCR4 driven colorectal cancer progression by Nef-M1 peptide. Proceedings of the 110th Annual Meeting of the American Association for Cancer Research; 2019 Mar 29-Apr 3; Atlanta, GA. Philadelphia (PA); AACR; 2019. Abstract nr 3879.

120.Lee RM, Hirano K, Anant S, et al. An alternatively spliced form of apobec-1 messenger RNA is overexpressed in human colon cancer. Gastroenterology 1998;115:1096-103.

121. Van Allen EM, Wagle N, Sucker A, et al. The Genetic Landscape of Clinical Resistance to RAF Inhibition in Metastatic Melanoma. Cancer Discov 2014;4:94-109.

122. Taylor BJ, Nik-Zainal S, Wu YL, et al. DNA deaminases induce break-associated mutation showers with implication of APOBEC3B and 3A in breast cancer kataegis. Elife 2013;2:e00534.

doi: $10.21037 / \operatorname{tgh} .2019 .12 .11$

Cite this article as: De Souza AL. Finding the hot spot: identifying immune sensitive gastrointestinal tumors. Transl Gastroenterol Hepatol 2020;5:48. 